\title{
Liquid Crystals: An Approach in Drug Delivery
}

\author{
P. RAJAK*, L. K. NATH AND B. BHUYAN \\ Department of Pharmaceutical Sciences, Dibrugarh University, Dibrugarh-786 004, India
}

Rajak, et al.: Liquid Crystals in Drug Delivery

Liquid crystal systems are attracting significant attention due to their unique microstructures and physicochemical properties. Liquid crystals are becoming the choicest system for $R$ and $D$ professionals in the formulation development sector. Drug delivery to desired biological targets can effectively be achieved using liquid crystals system. Liquid crystalline phases are formed via three routes, the molecular shape, microsegregation effects and chirality. The objective of the present review was to provide information on different forms of liquid crystal systems along with unique physicochemical properties and possible utilization of the unique physicochemical properties of the systems in pharmaceutical formulation development. This review summarized characterization of liquid crystal mesophases highlighting the multidisciplinary application of liquid crystals in drug delivery with respect to cubic and hexagonal phases.

Key words: Lyotropic liquid crystals, thermotropic liquid crystals, drug delivery, mesophases, cubic phase

A unique state of matter, called liquid crystal exists in case of certain substances that is located between solid and liquid state (fig. 1). Liquid crystals are typically elongated organic molecules with an uneven distribution of electrical charges along their axes (dipole). This give rise to a special physical characteristic to which liquid crystals owe their name, between the crystalline and liquid states. They possess both structural order and mobility. Liquid crystalline structures exhibit anisotropy, having optical direction and the characteristic properties of solids and liquids ${ }^{[1]}$. They exhibit a further state of aggregation, namely the liquid crystalline or mesophase indicating the unique structure intermediate between that of a true liquid and a solid crystal phase. Liquid crystals are found to be birefringent, due to their anisotropic nature. So they demonstrate double refraction (having two indices of refraction). When liquid crystalline structure is viewed under crossed Nicole prism of a polarizing microscope, intense colour bands and birefringence are seen. Anisometric molecular shape, associated with the polarizability is the basic requirement for the formation of liquid crystalline phases ${ }^{[2]}$. Even drugs that include organic acids or basic salts with anisometric molecular shape might fulfil the requirements for liquid crystal formation $^{[3]}$. On the basis of molecular structure, a compound may pass through one or many different liquid crystalline phases depending upon its order and symmetry, before transforming into a truly isotropic

*Address for correspondence E-mail: prakash@dibru.ac.in

January-February 2019 fluid (the liquid phase) $)^{[4]}$. Liquid crystals are categorized into two generic classes i.e. thermotropic and lyotropic mesophases ${ }^{[1,2]}$. Thermotropic liquid crystalline phases are formed by a change of temperature, whereas lyotropic phases are formed when mixed with aqueous phase. The phase transitions of thermotropic liquid crystal are temperature-dependent, while those of lyotropic liquid crystals depend on both temperature and concentration ${ }^{[5]}$.

Molecules in a crystal are highly ordered, while molecules in a liquid are free to diffuse in a random way. Thus, molecules in liquid crystal phases diffuse like the molecules of a liquid but they contain some degree of orderness ${ }^{[4]}$. Hexagonal and cubic mesophases are particularly of high interest in the drug delivery field due to their exceptional potential as drug vehicles ${ }^{[6,7]}$. They are highly investigated for their ability to control or sustain the release of both hydrophilic and hydrophobic drug molecules having wide range molecular weights. Drugs can be incorporated in these gel-like phases, additionally they have non-toxic, biodegradable and bioadhesive characteristics, which cause significant

This is an open access article distributed under the terms of the Creative Commons Attribution-NonCommercial-ShareAlike 3.0 License, which allows others to remix, tweak, and build upon the work non-commercially, as long as the author is credited and the new creations are licensed under the identical terms

Accepted 07 November 2018

Revised 18 September 2017

Received 24 February 2017

Indian J Pharm Sci 2019;81(1):11-21 
value addition in drug delivery ${ }^{[8]}$. These characteristics of hexagonal and cubic phases made them one of the favourable means for the researchers to deliver drug through different routes of administration e.g. buccal, gastrointestinal, intravenous, lung, nasal, oral, rectal and vaginal.

This review attempted to provide a comprehensive insight highlighting the multidisciplinary aspect of liquid crystal system. Owing to the wide applicability of liquid crystal system in different areas of science and technology, this review tried to investigate the current status of research to the application of cubic and hexagonal phases in the drug delivery context.

\section{Thermotropic liquid crystals:}

Most of the thermotropic liquid crystals are composed of rod-like molecules and classified into three types, nematic, smectic and cholesteric (fig. 2). They are formed on heating the crystalline solid or cooling the isotropic liquid. Nematic phase (thread-like) is the simplest liquid crystalline phase, where the molecules maintain long-range orientation. There exists no positional order. Liquid crystals used in electronic display are primarily of the nematic type. When viewed under a polarizing microscope the defect regions linking these domains appear as dark threads ${ }^{[9,10]}$. Smectic phase (soap-like) a name that was coined by Friedel from a Greek word, meaning 'grease or slime' ${ }^{[11]}$. The smectics structure is stratified as the molecules are arranged in layers with their long axes approximately normal to the plane of the layers with a well-defined interlayer spacing. Cholesteric phase is also known as chiral nematic liquid crystal. The arrangement of cholesteric phase can be described as a combination of the nematic and smectic, where some layers which resemble the smectic phase are incorporated in the nematic layers ${ }^{[12,13]}$. Due to the helical structure, it exhibits an interesting phenomenon like optical rotation, selective reflection and two-colour circular polarization ${ }^{[9]}$.

\section{Lyotropic liquid crystals:}

Lyotropic liquid crystals, briefly called lyotropics or lyomesophases are mixtures of amphiphilic molecules in a solvent at a given temperatures and relative concentration $^{[14]}$. The driving factors for the formation

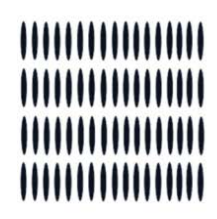

Crystal

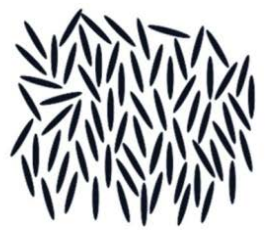

Liquid

Crystal
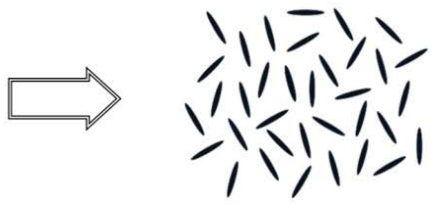

Liquid

Fig. 1: Formation of liquid crystal phase between crystal and liquid state of aggregation

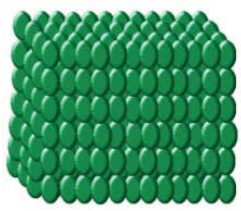

Crystal

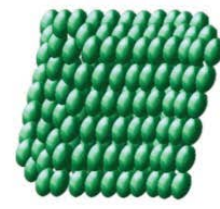

Smectic

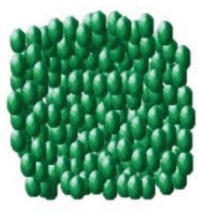

Nematic

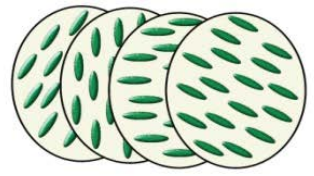

Cholesteric

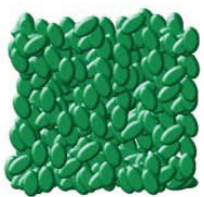

Isotropic

Liquid

Fig. 2: Thermotropic liquid crystals: crystal; smectic; nematic; cholesteric; isotropic liquid

Nematic phase (thread like) is the simplest liquid crystalline phase, where the molecules maintain long-range orientation. Smectics (soap-like) structure is stratified as the molecules are arranged in layers with their long axes approximately normal to the plane of the layers with a well-defined interlayer spacing. Cholesteric phase is also known as chiral nematic liquid crystal and the arrangement can be described as a combination of the nematic and smectic, where some layers which resemble the smectic phase are incorporated in the nematic layers 
of lyotropic mesophases are the temperature, the structure of the organic molecule, and the water/ amphiphile ratio ${ }^{[15,16]}$. Lyotropic liquid crystals are further classified as lamellar, hexagonal and cubic phase. Lyotropic liquid crystal systems of surfactant molecules can absorb water from the environment. Depending upon the water content of the environment, it triggers spontaneous phase-transitions resulting lamellar, hexagonal and cubic phases (fig. 3). Three properties of surfactant(s) are found to be affecting the formation of lyotropic liquid crystalline phase, these $\operatorname{are}^{[8]}$, the magnitude of the repulsive forces between adjacent head groups at the interface of surfactant and water, degree of contact between water and alkyl chain, and conformational disorders in the alkyl chains.

Lamellar phase or neat phase has double layers of surfactant molecule consisting of polar head groups, which protrudes in the intervening layers of aqueous interface. The hydrocarbon chains are in a dynamic disordered state that is similar to paraffin in the liquid state and the surfactant bilayers are separated by water layers ${ }^{[17,18]}$. A notable feature of the neat phase is its relative fluidity in spite of high surfactant content and thus the lamellae can glide easily over one another ${ }^{[19,20]}$.

Hexagonal phase or middle phase can be seen as an array of hexagonally close packed water layers sheltered by a surfactant monolayer. The long range order is two dimensional. Two types of hexagonal mesophase can be encountered: normal and reverse mesophase, respectively in aqueous and anhydrous organic solvents. In anhydrous organic solvents (low water content), the structure is reversed and the hydrophilic polar groups now form the inner core and are shielded from the non-aqueous environment by a layer of hydrocarbon chains. These are called reverse hexagonal phase or reverse middle phase. Also when large quantities on non-ionic surfactant are added to the system, a hexagonal structure and the solution containing reversed micelles may be formed. The hexagonal phase is much stiffer than the lamellar phase $^{[8,21,22]}$.

Cubic phase structure consists of continuous curved bilayers and a pair of interpenetrating non-intersecting aqueous channels separated by each other. Cubic phase has spherical packing and the ionic portion of the molecule on the surface of the sphere and the non-polar portion (water-insoluble) directed toward the centre of the sphere ${ }^{[19]}$. The interfacial area of cubic phase is comparatively larger than other phases. The unique microstructure of a cubic phase offers advantageous properties for controlled drug release. The close packing of the micelles accounts for the marked flow-resistance of the phase, which is stiffer than the mesophase $\mathrm{e}^{[8,22]}$. The cubic phase is formed spontaneously in excess water. Furthermore, the structure provides a slow release matrix for drugs of varying polarity and size because of its dual polarnonpolar nature. Additionally, release of the drug from within the cubic phase is controlled to certain degree because of its unique microstructure. Subsequently, the cubic phase displays bio adhesive properties. Thus, the structure is appropriate for gastrointestinal, pulmonary, nasal, oral, buccal, rectal, and vaginal drug delivery. As a whole, the cubic mesophase is stable in vitro ${ }^{[23]}$.

\section{Preparation of liquid crystal phase and its dispersion:}

Preparation of liquid crystal is very simple as compared to their dispersions. Liquid crystal can be prepared by intimately mixing lipid with aqueous phase by vortexing

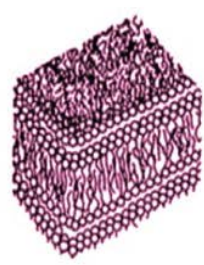

Lamellar

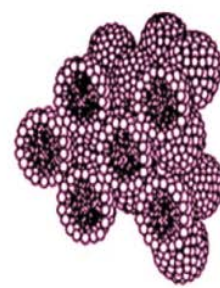

Cubic

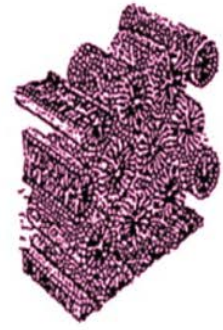

Hexagonal

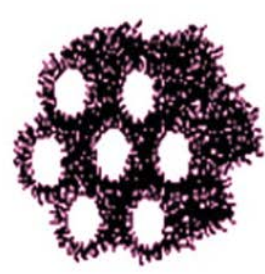

Reverse Hexagonal

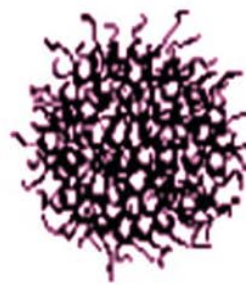

Reverse Cubic

Fig. 3: Lyotropic liquid crystal systems: lamellar; cubic; hexagonal; reverse hexagonal; reverse cubic Lamellar phase or neat phase has double layers of surfactant molecule consisting of polar head groups, which protrudes in the intervening layers of aqueous interface. Cubic phase structure consists of continuous curved bilayers and a pair of interpenetrating non-intersecting aqueous channels separated by each other. Cubic phase has spherical packing and the ionic portion of the molecule on the surface of the sphere and the non-polar portion (water-insoluble) directed toward the center of the sphere. Hexagonal phase or middle phase can be seen as an array of hexagonally close packed water layers sheltered by a surfactant monolayer. The long range order is two dimensional 
at high speed ${ }^{[12,13]}$. The vortexing can be repeated to achieve homogenous state. The mixture is equilibrated at room temperature for at least $48 \mathrm{~h}$ to obtain the liquid crystal. Depending upon the characteristics of the lipid, other additives may be added into the mixture or the method can be modified e.g. if more than one lipid is used, both the lipids are mixed and if required melted prior to vortex mixing with aqueous phase ${ }^{[3,8,22]}$. On the other hand preparation of liquid crystal dispersion is complex. Two techniques are used to prepare their dispersion. The first one is called the 'Top-Down' approach where at first the lipid and stabilizer is hydrated to self-assemble in a viscous bulk and then the bulk is dispersed into an aqueous solution by using high pressure homogenization and ultra-sonication. The second one is called as the 'Bottom-Up' approach, where the presence of hydrotrope plays the main role by creating liquid precursor and preventing liquid crystal formation at high concentration. Controlled addition of aqueous medium to the mixture leads to the formation of its dispersion. It is a dilution-based method and does not require any fragmentation procedure ${ }^{[13,8,18]}$.

\section{CHARACTERIZATION OF LIQUID CRYSTALLINE PHASES}

\section{X-ray diffraction:}

The most frequently used method for investigating liquid crystalline phases formed by surfactant and block copolymer systems is no doubt the X-ray diffraction. With X-ray scattering experiments, characteristic interferences are generated from an ordered microstructure. There are two methods to detect interferences utilizing position-sensitive detectors/scintillation counters i.e. registration of X-ray counts and film detection. Due to the presence of longrange structural order in liquid crystals, generation of diffraction patterns are possible as electromagnetic radiation of a suitable wavelength will result in interaction.

Utilizing small-angle X-ray diffraction (SAXD) and wide-angle X-ray diffraction, long and short range orders can be registered respectively. In the characterization of liquid crystals SAXD is important tool for determining diffraction pattern (Table 1$)^{[24]}$.

\section{Nuclear magnetic resonance (NMR) spectroscopy:}

NMR spectroscopy offers a range of possibilities for investigating liquid crystalline phases. Deuterium $\left({ }^{2} \mathrm{H}\right)$ NMR spectroscopy offers a powerful technique for
TABLE1: RELATION BETWEEN THE FIRST REFLECTIONS FOR DIFFERENT LIQUID CRYSTALLINE PHASES

\begin{tabular}{|c|c|}
\hline Cubic & 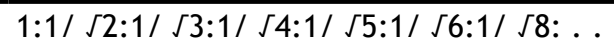 \\
\hline Lamellar & $1: 1 / 2: 1 / 3: 1 / 4:$ \\
\hline Hexagonal & $1: 1 / \sqrt{ } 3: 1 / \sqrt{ } 4: 1 / \sqrt{ } 7:$. \\
\hline
\end{tabular}

investigating phase equilibrium, since the occurrence of different phases can be straight forwardly identified with this method. The basis for this method is that isotropic phase yields a narrow singlet resonance signal of quadrupolar nuclei such as ${ }^{2} \mathrm{H}$, whereas anisotropic phases result in so-called quadrupolar splitting, thereby yielding a doublet resonance signal. Furthermore, anisotropic phases may be separated through the magnitude of the splitting. From this, not only the nature of the phase in the case of a single-phase system can be determined, but the composition of a multiphase system may also be determined.

\section{Polarizing microscopy:}

It is very simple and vivid method. As a result of their molecular ordering, anisotropic liquid crystalline phases, such as the hexagonal, the lamellar, and the reversed hexagonal phases, are optically birefringent. Polarized light microscope can be used for the study of the phases. In this microscope one polarizer is mounted above and the other below in cross position of the objective providing plane polarized light at right angle, whereby restricting the passage of polarized light from the below to the above polarizer of the objective. There is a possibility of observing turquoise and yellow textures with liquid crystalline material due to small deviations of the absorbed wavelengths. The lamellar phase usually yields mosaic patterns under the polarizing microscope, whereas the hexagonal phases normally show nongeometric textures. The occurrence of crystals may also be identified by this method. Because of the non-birefringent nature of the micellar and reversed micellar solutions and cubic phase dark background will be observed. The smectic mesophases of the thermotropic liquid crystal show a variety of textures but resemble the fan-shaped texture of the lyotropic hexagonal mesophase ${ }^{[25,26]}$. Although polarized optical microscopy is one of the powerful tool used to identify liquid crystal phases, due to submicron scale of liquid crystal mesophases this method may fall short. On the other hand interaction of other imaging techniques may also causes degradation of the sample $\mathrm{e}^{[27]}$. 


\section{Transmission electron microscopy:}

Because of the high magnification power of the electron microscope, the microstructure of liquid crystals can be visualized. Special techniques are adopted for preparation of aqueous samples as the samples tend to succumb in the high vacuum of an electron microscope that is responsible for inherent changes in the microstructure. The freeze-fracture technique has proven to be successful in this regard. For this purpose, a replica of the sample is produced and viewed in the electron microscope. To preserve the original microstructure of the sample during the replication, the first step is to shock freeze the sample. For high freezing rates up to $105-106 \mathrm{~K} / \mathrm{s}$, the sample is sandwiched as a thin layer between two gold plates and then shock frozen with nitrogen-cooled liquid propane at $-196^{\circ}$ or slush nitrogen at $-210^{\circ}[28]$. The application of pressure ensures minimal crystallization of the water within the sample to preserve the structure as best as possible ${ }^{[27]}$.

\section{Differential scanning calorimetry:}

Phase transitions occur with changes in the energy content of the respective system. Depending on the transition type i.e. consumption of energy due to melting of solid or emission of energy due to recrystallization, endothermic or exothermic signals can be observed. The consumption of energy gradually decreases from crystalline to amorphous; between the transition from crystalline to amorphous phase, liquid crystalline phase occurs and the energy consumption among liquid crystalline phase transitions consume comparatively lesser energy ${ }^{[28]}$. Necessary care has to be taken about the sensitivity and detection limit of the measuring device. Change of baseline slope with a change in specific heat capacity is an indicator of entropy change during the phase transition. Therefore the phase transitions of liquid crystalline polymers result from entropic reasons are considered as transitions of the second order ${ }^{[29]}$. These are usually called glass transitions. They may be overlaid from an enthalpic effect, so their detection may be complicated.

\section{Rheology:}

Rheological properties of various degrees are shown by different forms of liquid crystals ${ }^{[30]}$. With an increase in the microstructural organization of the liquid crystal, its consistency increases and the flow behaviour becomes more viscous. The coefficient of dynamic viscosity Z, although a criterion for the viscosity of ideal viscous flow behaviour (Newtonian systems), hexagonal, and cubic liquid crystals shows comparatively higher viscosity than lamellar phase. Moreover, plastic flow behaviour (non-Newtonian) is observed in cubic and hexagonal crystals but pseudo plastic flow behaviour (non-Newtonian) is observed in lamellar phase; which is the key feature to differentiate between these two types $^{[31]}$. For thermotropic liquid crystals, the viscosity increases in the following sequence: nematic $<$ smectic $\mathrm{A}<$ smectic $\mathrm{C}$.

\section{Vesicle size determination:}

Vesicle size is an important parameter in not only inprocess control but also quality assurance in particular as the particle size and particle size distribution is a major determinant of the physical stability of vesicle dispersion $^{[32]}$. An appropriate and particularly quick method is laser light scattering (for particle size) or diffraction (for particle size distribution). Laser light diffraction can be applied for $>1 \mathrm{~mm}$ particles; according to the diffraction theory of Fraunhofer, this refers to the proportionality between the intensity of the diffraction and the square of the particle diameter.

Fraunhofer's and Rayleigh's theories are just an approximations of Mie's theory, where scattering angle, absorption, size and refractive indices of particles and the dispersion medium are the major factors affecting the scattering intensity ${ }^{[3]}$. Photon correlation spectroscopy can be a good tool for determination of mesophase with particle sizes of $<200 \mathrm{~nm}$ up to $1 \mathrm{~mm}$.

\section{APPLICATION OF LIQUID CRYSTALS IN DRUG DELIVERY}

The ability of LCs to include a vast array of molecules from lipophilic to hydrophilic irrespective of molecular dimensions is one of the significant parameters that are of interest ${ }^{[33]}$. Arsphenamin was the first drug substance with thermotropic mesomorphism to be therapeutically used as Salvarsan during the first half of the $19^{\text {th }}$ century. The drug is effective against microorganisms and offered therapy for venereal diseases such as syphilis. Today, it has been replaced by antibiotics with less serious side effects.

The stability and resemblance with skin structure make lamellar phases suitable for the development of controlled-release drug delivery systems. The watersoluble, oil-soluble and amphiphilic drugs can easily be incorporated within the peculiar arrangement of lipid bilayers and aqueous channels of lamellar 
phase $^{[34]}$. The drug candidate can permeate through the hydrophilic regions between the lamellae, trapped inside lipid bilayer and move both between and across the lamellae ${ }^{[35]}$. However the whole process depends upon the solubility/affinity of the drug molecule.

A lamellar system consisting Brij 96 (poly-oxyethylene10-oleyl ether) with water, liquid petrolatum and glycerol incorporated with ephedrine hydrochloride or tenoxicam, the former being a hydrophilic drug and the latter being hydrophobic drug was reported by Makai et al. ${ }^{[36]}$. The study detected an increase in interlamellar distance inferring that the drugs were partly located between the lamellar spaces and partly located at the amphiphilic surfactant molecules.

\section{Application of cubic phases:}

Cubic phase consists of curved lipid bilayers extending in three dimensions and separated by two congruent networks of water channels ${ }^{[37]}$. Cubic phase has a stiff, transparent, gel-like appearance and possess bioadhesive properties. Biodegradability is another important feature with regards to drug delivery ${ }^{[38]}$. Interfacial area of cubic phase is about $400 \mathrm{~m}^{2}$ per $\mathrm{g}$ and the pore size of fully swollen cubic phases is about $5 \mathrm{~nm}^{[39,40]}$. In cubic phases the activity of water is minimum as well as it poses bio membrane like structures, which makes this system suitable for delivering protein molecules by protecting from external factors, thereby avoiding inactivation of protein molecules ${ }^{[37]}$.

Glyceryl monooleate (GMO)-water system, an amphiphilic lipid, forms various liquid crystalline phases in contact with water. Bioavailability of poorly water-soluble drugs can be greatly enhanced with the incorporation of GMO in the delivery system. The drug delivery effectiveness of a binary GMO-water liquid crystalline phase composition is GMO:water ratiodependent. Binary liquid crystalline phase systems are categorized as having either relatively high or low water content. A binary composition having a weight ratio less than about 1:1 GMO to water is not useful as it separates into aqueous and liquid crystalline phases thus rendering the system ineffective ${ }^{[12,8]}$. The Pluronic F127 system has a high solubilizing capacity and is generally considered to be relatively non-toxic. In aqueous solution, at concentrations greater than $20 \% \mathrm{w} / \mathrm{w}, \mathrm{F} 127$ is transformed upon heating from a low viscosity transparent (micellar) solution at room temperature to a solid clear gel (cubic phase) at body temperature ${ }^{[41]}$.
Lipids forming non-lamellar structures, such as monoolein, are now getting incorporated in novel protein biochip developments. As cubic lipid phases are biocompatible and digestible, these are being used for the development for controlled-release and delivery of proteins, vitamins and small drugs in pharmacological applications ${ }^{[9]}$.

Cubic phase particles (cubosomes) are submicron particles of bicontinuous cubic phases for incorporation of lipophilic or amphiphilic active ingredient. Cubosomes addresses different challenges in oral delivery of many promising compounds that has poor aqueous solubility, poor absorption, and large molecular size. The presence of liquid crystal matrix provides a good option to control the release of drug form the system to obtain optimal delivery profile along with temporary protection of sore and sensitive skin. Cubosomes with metronidazole are available commercially to treat periodontal diseases based on combination of triglyceride-monoolein mixtures. The oral administration of insulin loaded into GMO cubic phase also has been successfully investigated ${ }^{[42]}$.

Kuntsche et al. ${ }^{[43]}$ reported that supercooled smectic cholesteryl myristate nanoparticles could be loaded with different model drugs without loss of the smectic state structure. Drugs of lower melting points such as ibuprofen, etomidate and miconazole were incorporated in the dispersions at a concentration of $10 \% \mathrm{w} / \mathrm{w}$, whereas progesterone with a higher melting point could only be dissolved in the lipid melt at a concentration of $1 \% \mathrm{w} / \mathrm{w}$ at appropriate dissolution times.

Kuntsche et al. ${ }^{[44]}$ further studied the permeation of the model drug corticosterone using different lipid nanoparticles. They reported that smectic nanoparticles seem to have no influence on the corticosterone permeation, and similar results were obtained for human and the cell culture epidermis. Only cubic nanoparticles enhanced drug permeation distinctly and the enhancing effect was 7 fold higher in human epidermis than in the rat epidermal keratinocytes organotypic culture. However, the variation of the permeability coefficients was very high in human skin for smectic and cubic nanoparticles especially between the skin samples obtained from different donors.

Nag et al. synthesized nanoparticles incorporating the water-insoluble model dye cargo, 3,3'-dioctadecyloxacarbocyanine perchlorate (DiO) into the hydrophobic liquid crystal nanoparticle (LCNP) core (DiO-LCNP-PEG-Chol). The LCNP platform 
was found to be efficient vehicle for the combined membrane-targeted delivery and physicochemical modulation of molecular cargos using lipid raftmediated tethering ${ }^{[45]}$.

Sallam et al. formulated liquid crystal system containing $10 \%$ PG, $5 \%$ water and $65 \%$ GMO that contains $7 \%$ EC loaded with $20 \%$ metronidazole. They found the formulation to be mucoadhesive, easily injectable at room temperature, and followed Fickian diffusion release mechanism ${ }^{[46]}$.

Swarnakar et al. prepared phytantriol (PHY)-based doxorubicin-LCNPs using the hydrotrope method. The formulation exhibited Pn $3 \mathrm{~m}$ type cubic structure and possessed significantly higher cell cytotoxicity (16.23-fold) against MCF-7 cell lines as compared to free drug. Doxorubicin-induced cardiotoxicity was also found to be significantly lower in case of doxorubicinLCNPs as compared to clinical formulations ${ }^{[4]]}$.

Hongbing et al. succeeded in developing a protocol for surface engineering of PEGylated cubosomes with functional molecules, mediating S14G-HN transport into the brain for ameliorating learning impairment in rats. Where most of the peptides and proteins with considerable potential in the treatment of brain diseases are susceptible to enzymes in vivo and hold a poor ability to cross $\mathrm{BBB}$, the developed cubosomes showed potential to serve as carrier system ${ }^{[48]}$.

Shah et al. incorporated seratiopeptidase (STP) in cubic phase carrier utilizing GMO. Magnesium trisilicate was found to improve stability of STP. They demonstrated that the in situ cubic phase transforming system can be used as a carrier for protein molecules by altering the microenvironment and water uptake by the matrix ${ }^{[49]}$.

Sung et al. reported that monoolein cubosomes can effectively damp the fluctuation I time-dependent size and zeta potential KIOM-C. At all $\mathrm{pHs}$ tested ( $\mathrm{pH}$ 2.0-10.0), baicalin, a major component of KIOM-C, was found to be chemically stable for $48 \mathrm{~h}$ at a room temperature. With the aid of cubosome, baicalin suspension remained homogenous without significant sedimentation for $180 \mathrm{~min}^{[50]}$. Bei et al. enumerated different factors that may influence the loading and encapsulation efficiency of dacarbazineloaded cubosomes ${ }^{[51]}$.

Jin et al. developed cubic nanoparticles to increase the absorption of 20(S)-protopanaxadiol in which piperine was loaded for inhibition of metabolism. This novel carrier containing piperine showed an increase in the absorption and inhibition of metabolism, which in turn increases the bioavailability of 20(S)-protopanaxadiol. This system may be further investigated for anticancer drugs that are extensively metabolized and have low absorption $^{[52]}$.

Zhiwen et al. prepared cubosomes from PHY containing amphotericin B as a formulation for oral delivery. Cubosomes having nanometer-sized particles were obtained under homogenization at 1200 bar for 9 cycles $^{[53]}$. Jain et al. demonstrated GMO-based PEGylated drug delivery system of paclitaxel, prepared by optimizing several parameters in order to discover its potential in anticancer therapy ${ }^{[54]}$.

Nguyen et al. worked on LCNPs to prolong the absorption of a poorly water-soluble drug cinnarizine (CZ) after oral administration. Cubic liquid crystalline particles developed by utilizing PHY were found to be prolonging the absorption of $\mathrm{CZ}$ more than $48 \mathrm{~h}$ after administering orally to rats ${ }^{[55]}$.

Thapa et al. found that a liquid crystalline nanoparticle reveals unique structures, which can be used in the delivery of various active pharmaceutical ingredients. The influence of saturated fatty acids on tacrolimusloaded liquid crystalline nanoparticles stabilized with poloxamer 407 was also investigated ${ }^{[56]}$.

Lee et al. reported the in vitro sustained release behaviour of a number of model hydrophilic drugs with various molecular weights $\left({ }^{14} \mathrm{C}\right.$-glucose, Allura Red, and fluorescein isothiocyanate dextrans FD-4, FD-20, and FD-70) in liquid crystalline matrixes prepared from GMO and $\mathrm{PHY}^{[57]}$. Nguyen et al. reported the sustained absorption of a poorly water-soluble drug (CZ) from PHY-based cubosomes after oral administration. The plasma concentration of PHY-based cubosomes was found to be $21.5 \pm 1.5 \mathrm{ng} / \mathrm{ml}$ within 12 and $48 \mathrm{~h}$, while the plasma concentrations felt below the quantification limit after $24 \mathrm{~h}$ when treated with suspensions or GMObased cubosomes ${ }^{[55]}$.

Esposito et al. studied in vitro diffusion of indomethacin from carbomer-indomethacin-loaded cubosomes. The performance of cubosomes as sustained percutaneous delivery systems was found to be satisfactory as the flux of indomethacin from cubosomes was significantly lower than carbomer blank cubosomes loaded with free indomethacin and carbomer with an indomethacin water suspension ${ }^{[58]}$.

Chen et al. reported a cyclosporine A-loaded GMO/ F127 cubosome system to reduce ocular irritancy 
showing little or no ocular irritancy. The formulation was found to be safe to use with no ocular damage or clinically abnormal signs in cornea, conjunctiva, or iris $^{[59]}$. Sadhale and Shah investigated the capability of cubic phase gel to protect insulin from agitation-induced aggregation. The agitated insulin in cubic phase gels was almost unaffected for 2 mo at $37^{\circ}$ as compared to insulin in solution, which showed aggregation and precipitation after $8 \mathrm{~d}^{[60,61]}$.

Sadhale and Shah also prepared GMO cubic phase gel containing cefazolin and cefuroxime and assessed their stability at two different concentrations (200 and $50 \mu \mathrm{g} / \mathrm{g}$ ), at 22 and $37^{\circ}$. The degradation of cefazolin at lower concentration was 3 and 18 fold slower in cubic phase gel than that in solutions at 22 and $37^{\circ}$, respectively ${ }^{[62]}$. Spillmann et al. established multifunctional nanoparticle-based delivery system, which provides efficient delivery and allows intracellular fate tracking ${ }^{[63]}$.

\section{Application of hexagonal phases:}

Hexagonal mesophases are closed and extended micellar columnar structures having two-dimensional long range order. There is no direct contact between water inside and outside the hexagonal phases. The dispersed reversed hexagonal particles are called as hexosomes. The hexagonal mesophases composed of oleyl glycerate (OG, 2,3-dihydroxypropionic acid octadec-9-enyl ester) and phytanyl glycerate (PG, 2,3-dihydroxypropionic acid 3,7,11,15-tetramethylhexadecyl ester) have shown great potential in drug delivery. This system facilitates incorporation of both hydrophilic and lipophilic drugs as well as amphiphilic drugs, entrapping hydrophilic drugs in the internal water domain and lipophilic drugs within the lipid domain whereas amphiphilic drugs in the interface.

Nilsson et al. reported a highly efficient method for the radiolabeling of PHY/oleic acid (OA)-based hexosomes based on the surface chelation of technetium-99m $((99 \mathrm{~m}) \mathrm{Tc})$ to preformed hexosomes using the polyamine 1, 12-diamino-3,6,9triazododecane (SpmTrien) as chelating agent. They also concluded that the developed (99m) Tc labelling method could serve as a useful tool for investigating and imaging the in vivo performance of cubosomal and hexosomal drug nanocarriers ${ }^{[64]}$.

Boyd et al. synthesized a new class of materials with glycerate headgroups to form lyotropic liquid crystal. The OG and PG-based hexagonal phase matrices were used as carrier for paclitaxel, irinotecan, glucose, histidine, and octreotide. The study revealed that the releases of all the formulations were following Higuchi kinetics confirming their ability to use as carrier in sustained release drug delivery ${ }^{[21]}$.

Boyd et al. stated that the OG-based hexagonal phase improved the oral bioavailability of $\mathrm{CZ}$ up to 3.5 and 3 times than that of the control suspension and GMObased cubic phase, respectively ${ }^{[65]}$. Boyd et al. also reported that the OG-based hexosomes can be used to improve stability of irinotecan, a highly effective anticancer drug, which displays a pH-dependent equilibrium between its active lactone and inactive carboxylate forms, with rapid conversion to the carboxylate form occurring at a neutral $\mathrm{pH}^{[66]}$.

Lopes et al. reported that vitamin $\mathrm{K}$ delivery to the stratum corneum was increased by 2 times and delivery to the epidermis and dermis was increased by 2 to 3.7 times than that in a vaseline control solution when incorporated into GMO-based hexagonal phase gel (GMO/vitamin $\mathrm{K} /$ water at $77.5 / 2.5 / 20, \mathrm{w} / \mathrm{w} / \mathrm{w})$ or GMO-based hexosomes (GMO/vitamin K/F127/water at $15 / 2.5 / 0.9 / 81.6, \mathrm{w} / \mathrm{w} / \mathrm{w} / \mathrm{w})^{[67]}$.

Swarnakar et al. investigated the penetration mechanism of hexosome system as a carrier of progesterone for mucosal delivery. The flux of progesterone-loaded hexosomes to the excised rabbit buccal mucosa was 5 times higher than that of a progesterone-loaded gel and nearly 4 times higher than a plain progesterone suspension $^{[68]}$.

Fong et al. reported a themoresponsive liquid crystalline system to deliver glucose (hydrophilic drug) through subcutaneous route. The in vivo study of the externally regulated themoresponsive system showed slow release from hexagonal phase when subcutaneously injected at physiological temperature ${ }^{[69]}$.

Lee et al. reported fivefold enhancement of bioavailability, sustained release, and liver-specific drug delivery of prepared LCNPs containing hydrophobic peptide-based drugs as compared to a host-guest complex formulation. The LCNPs were prepared by using an energy efficient phase-inversion temperature method ${ }^{[70]}$.

Swarnakar et al. prepared CoQ10-loaded hexosomes from glyceryl monooleate (GLCQ) and phytantriol (PHYLCQ) and investigated the implications of the lipase digestibility of hexosomes on the oral bioavailability, in vivo antioxidant potential, and 
IVIVR relationship. They observed 9.1 and 10.67 fold increase in Caco-2 cell uptake in the case of GLCQ and PHYLCQ, respectively. Bioavailability was increased by 7.09 and 8.67 fold in the case of GLCQ and PLCQ, respectively. The developed formulation found to have great potential in improving the oral bioavailability of difficult-to-deliver drugs such as CoQ10 ${ }^{[71]}$.

\section{Application of cubic-hexagonal phases and their inter-conversion:}

Many researchers also reported the inter-conversion between different phases of liquid crystal influenced by the temperature, pressure and $\mathrm{pH}$. They have utilized the inter-conversion ability of the system to either target the delivery of drug molecules or to increase stability and bioavailability in different physiological conditions.

Okawara et al. prepared diosgenin LCs and investigated the interaction between $\mathrm{LC}$ and $\beta-\mathrm{CD}$ in order to improve its bioavailability of diosgenin. The SAXD patterns showed formation hexagonal and cubic phases of GMO and PHY LC, respectively. The oral bioavailability of LCs prepared by GMO was significantly enhanced than diosgenin alone, which was further improved with the combination of $\mathrm{LC}$ and $\beta-\mathrm{CD}$ than $\mathrm{LC}$ and water ${ }^{[72]}$.

Yaghmur et al. prepared inverted type dis-continuous cubic phase $(\mathrm{Fd} 3 \mathrm{~m})$ and inverted type hexagonal phase $\left(\mathrm{H}_{2}\right)$ and stability/inter-conversion under pressure and temperature was investigated. Under ambient temperature phase transition from $\mathrm{Fd} 3 \mathrm{~m}$ to $\mathrm{H}_{2}$ was reported and opposite trend was observed when temperature was increased at isobaric condition causing transition from $\mathrm{H}_{2}$ to $\mathrm{Fd} 3 \mathrm{~m}^{[73]}$.

Sallam et al. indicated that the acidic environment favours the formation of hexagonal phase by observing the formation of reversed hexagonal phase at a lower temperature in simulated gastric fluid than that in simulated intestinal fluid or water ${ }^{[74]}$. Negrini and Mezzenga reported that a system composed of monolinolein and linoleic acids $(97 / 3, \mathrm{w} / \mathrm{w})$ was able to reversibly change from a reverse bicontinuous cubic phase to a reverse hexagonal phase, when the $\mathrm{pH}$ was dropped from 7 to $2^{[75]}$.

Negrini et al. investigated various liquid crystal matrices made of monolinolein+drug+linoleic acid+excess water in different $\mathrm{pH}$ conditions and their release behaviour. The matrices were in a cubic phase in $\mathrm{pH} 7$ and hexagonal in $\mathrm{pH} 2^{[76]}$. Yaghmur et al. studied the effect of $\mathrm{pH}$ on the phase structures of GMO-based liquid crystals containing bupivacaine. Different self-assembled structures were formed by increasing the $\mathrm{pH}$ from 6.0 to 7.4, which induced the structural transition of bicontinuous cubic phase to reverse hexagonal phase ${ }^{[77,78]}$.

Yuli and Garti studied the effect of triglycerides with various chain lengths into the binary GMO/water system. The study revealed the phase transition from lamellar or cubic phases to hexagonal structure at room temperature as the triglyceride gets solubilized in the system $^{[79]}$.

This review emphasized the characterization and application of liquid crystalline systems as drug carrier, highlighting its applications in drug delivery. Drug delivery to desired biological targets can effectively be achieved using liquid crystals technique as they respond to both $\mathrm{pH}$ and temperature depending upon the type of liquid crystal systems utilized for the delivery of drug molecules. Due to its wide array of drug loading irrespective of its hydrophobicity or hydrophilicity, it can be utilized for formulating any kind of formulation intended for delivery via various routes of administration. Hexagonal and cubic mesophases are particularly of high interest due to their exceptional potential as drug vehicles. This technique of drug loading is becoming one of the most promising techniques and requires extensive research to widen its spectra in the formulation development arena.

\section{Conflicts of interest:}

All authors declare no conflict of interest.

\section{REFERENCES}

1. Martin AN, Sinko PJ. Martin's Physical Pharmacy and Pharmaceutical Sciences. Physical Chemical and Biopharmaceutical Principles in the Pharmaceutical Sciences. 6th ed. Baltimore, Philadelphia: Lippincott Williams \& Wilkins; 2011. p. 112-43.

2. Lancelot A, Sierra T, Serrano JL. Nanostructured liquid crystalline particles for drug delivery. Expert Opin Drug Deliv 2014;11(4):547-64.

3. Muller-Goymann CC. Physicochemical characterization of colloidal drug delivery systems such as reverse micelles, vesicles, liquid crystals and nanoparticles for topical administration. Eur J Pharm Biopharm 2004;58:343-56.

4. Rochow TG. Compound Microscopes Using Reflected Light. In: Rochow TG, Tucker PA, editors. Introduction to Microscopy by Means of Light, Electrons, X Rays, or Acoustics. Philadelphia, Pennsylvania: Springer US; 1994. p. 56-98.

5. Clercq KDE. Formulation and characterization of liquid 
crystal platforms for the treatment of periodontal diseases [dissertation]. Belgium: Ghent University; 2012.

6. Li Y, Dong C, Cun D, Liu J, Xiang R, Fang L. Lamellar liquid crystal improves the skin retention of 3-o. AAPS PharmSciTech 2016;17(3):767-77.

7. Calixto GM, Bernegossi J, de Freitas LM, Fontana CR, Chorilli M. Nanotechnology-based drug delivery systems for photodynamic therapy of cancer: a review. Molecules 2016;21: 342-50.

8. Guo C, Wang J, Cao F, Lee RJ, Zhai G. Lyotropic liquid crystal systems in drug delivery. Drug Discov Today 2010;15(2324):1032-40.

9. Hussain A, Pina AS, Roque ACA. Bio-recognition and detection using liquid crystals. Biosens Bioelectron 2009;25:1-8.

10. Kawamoto $\mathrm{H}$. The history of liquid crystal display. Proc IEEE 2002;90(4):460-99.

11. Oswald P, Pieranski P. Nematic and cholesteric liquid crystals: concepts and physical properties. Boca Raton, Florida, United States: CRC Press; 2005. p. 17-20.

12. Kelker H, Hatz R. Handbook of Liquid Crystals. Weinheim, Germany: Verlag Chemie; 1980. p. 121-34.

13. Stevenson CL, Bennett DB, Lechuga-Ballesteros D. Pharmaceutical liquid crystals: the relevance of partially ordered systems. J Pharm Sci 2005;94:1861-80.

14. Jakli A, Saupe A. One- and Two-Dimensional Fluids: Physical Properties of Smectic, Lamellar and Columnar Liquid Crystals. Boca Raton, Florida, United States: CRC Press; 2006. p. 256-70.

15. Attwood D. Surfactant Systems: Their Chemistry, Pharmacy and Biology. Netherlands: Springer; 1983. p. 121-45.

16. Siddig MA, Radiman S. Structure of cubic phases in ternary systems glucopone/water/hydrocarbon. Colloids Surf A Physicochem Eng Asp 2004;236(1-3):57-67.

17. Carvalho FC, Barbi MS, Sarmento VH, Chiavacci LA, Netto FM, Gremião MP. Surfactant systems for nasal zidovudine delivery: structural, rheological and mucoadhesive properties. J Pharm Pharmacol 2010;62(4):430-9.

18. Rosevear FB. Liquid crystals: the mesomorphic phases of surfactant compositions. J Soc Cosmet Chem 1968;19:581-94.

19. Shah MH, Biradar SV, Paradkar AR. Spray dried glyceryl monooleate-magnesium trisilicate dry powder as cubic phase precursor. Int J Pharm 2006;323:18-26.

20. Mohammady SZ, PouzotM, Mezzenga R. Oleoylethanolamidebased Lyotropic Liquid Crystals as Vehicles for Drug Delivery of Amino Acids in Aqueous environment. Biophys J 2009;96:1537-46.

21. Boyd BJ, Whittaker DV, Khoo SM, Davey G. Lyotropic liquid crystalline phases formed from glycerate surfactants as sustained release drug delivery systems. Int $\mathrm{J}$ Pharm 2006;309:218-26.

22. Fraser S, Separovic F, Polyzos A. Cubic phases of ternary amphiphile-water systems. Eur Biophys J 2009;39(1):83-90.

23. Clogston J, Caffrey M. Controlling release from the lipidic cubic phase. Amino acids, peptides, proteins and nucleic acids. J Control Release 2005;107(1):97-111.

24. Fontell K. Liquid crystals and plastic crystals. In: Gray GW, Winsor PA, editors. Liquid crystals and plastic crystals: physico-chemical properties and methods of investigation. Chichester: Ellis Horwood Publishers; 1974. p. 80-109.

25. Khoo IC. Liquid Crystals: Physical Properties and Nonlinear
Optical Phenomena. New York: Wiley-Interscience; 1987. p. 214-85.

26. de Gennes PG, Prost J. The Physics of Liquid Crystals. Oxford, United Kingdom: Oxford University Press; 1993. p. 25-6, 175-9.

27. Gao M, Kim YK, Zhang C, Borshch V, Zhou S, Park HS, et al. Direct observation of liquid crystals using cryo-TEM: specimen preparation and low-dose imaging. Microsc Res Tech 2014;77(10):754-72.

28. Burylov SV, Raikher YL. Orientation of a solid particle embedded in a monodomain nematic liquid crystal. Phys Rev E Stat Phys Plasmas Fluids Relat Interdiscip Topics 1994;50:358-67.

29. Murgia S, Bonacchi S, Falchi AM, Lampis S, Lippolis V, Meli V, et al. Drug-loaded fluorescent cubosomes: versatile nanoparticles for potential theranostic applications. Langmuir 2013;29(22):6673-9.

30. Monteiro N, Martins A, Reis RL, Neves NM. Liposomes in tissue engineering and regenerative medicine. J R Soc Interface 2014;11(101):1-24.

31. Roux D, Nallet F, Diat O. Rheology of lyotropic lamellar phases. Europhys Lett 1993;15:53-8.

32. Bertelsen BM, Korsholm KS, Rose F, Nordly P, Franzyk H, Andersen $\mathrm{P}$, et al. The supramolecular structure is decisive for the immunostimulatory properties of synthetic analogues of a mycobacterial lipid in vitro. RSC Adv 2013;3:20673-83.

33. Malmsten M. Surfactants and Polymers in drug delivery. New York: Marcel Dekker Inc; 2006. p. 116-212.

34. Wahlgren S, Lindstrom AL, Friberg SE. Liquid crystals as a potential ointment vehicle. J Pharm Sci 1984;73:1484-6.

35. Geraghty PB, Attwood D, Collett JH, Dandiker Y. In vitro release of some antimuscarinic drugs from monoolein / water lyotropic liquid crystalline gels. Pharm Res 1996;13:1265-71.

36. Makai M, Csanyi E, Nemeth Z, Palinkas J, Eros I. Structure and drug release of lamellar liquid crystals containing glycerol. Int J Pharm 2003;256:95-107.

37. Shah JC, Sadhale Y, Chilukuri DM. Cubic phase gels as drug delivery systems. Adv Drug Deliv Rev 2001;47:229-50.

38. Wallin R, Arnebrant T. The activity of lipase at the cubic liquid crystalline phase/water interface. J Colloid Interface Sci 1994;164:16-20.

39. Engstrom E, Lindahl L, Wallin R, Engblom J. A study of polar lipid drug carrier system undergoing a thermo reversible lamellar to cubic phase transformation. Int J Pharm 1992;86:137-45.

40. Wyatt D, Dorschel D. A cubic-phase delivery system composed of glyceryl monooleate and water for sustained release of water-soluble drugs. PharmTech 1992;16:116-30.

41. Lawrence MJ. Surfactant systems: their use in drug delivery. Chem Soc Rev 1994;23:417-23.

42. Chung H, Kim J, Um YJ, Kwon IC, Jeong SY. Self-assembled "nanocubicle" as a carrier for peroral insulin delivery. Diabetologia 2002;45:448-51.

43. Kuntsche J, Westesen K, Drechsler M, Koch MH, Bunjes H. Supercooled smectic nanoparticles: a potential novel carrier system for poorly water soluble drugs. Pharm Res 2004;21:1834-43.

44. Kuntsche J, Bunjes H, Fahr A, Pappinen S, Ronkko S, Suhonen $\mathrm{M}$, et al. Interaction of lipid nanoparticles with human epidermis and an organotypic cell culture model. Int J Pharm 2008;354:180-95.

45. Nag OK, Naciri J, Oh E, Spillmann CM, Delehanty JB. 
Lipid raft-mediated membrane tethering and delivery of hydrophobic cargos from liquid crystal-based nano-carriers. Bioconjug Chem 2016;27(4):982-93.

46. Sallam AL, Hamudi FF, Khalil EA. Effect of ethyl cellulose and propylene glycol on the controlled-release performance of glyceryl monooleate-metronidazole periodontal gel. Pharm Dev Technol 2015;20(2):159-68.

47. Swarnakar NK, Thanki K, Jain S. Bicontinuous cubic liquid crystalline nanoparticles for oral delivery of doxorubicin: implications on bioavailability, therapeutic efficacy, and cardio toxicity. Pharm Res 2014;31(5):1219-38.

48. Hongbing W, Jianxu L, Qizhi Z, Xiluan Y, Liangran G, Xiaoling $\mathrm{G}$, et al. A novel small odorranalctin-bearing cubosomes: Preparation brain delivery and pharmacodynamics study on amyloid- $\beta 25-35$ treated rats following intranasal administration. Eur J Pharm Biopharm 2012;80:368-78.

49. Shah MH, Paradkar A. Cubic liquid crystalline Glyceryl monooleate matrices for oral delivery of enzyme. Int J Pharm 2005;294:161-71.

50. Sung KH, Jin YM, Jin-Chul K. Preparation of iron oxide nanoparticles within monoolein cubic phase. Ind Eng Chem Res 2012;18:1977-82.

51. Bei D, Marszalek J, Youan B-BC. Formulation of dicarbazineloaded Cubosomes-Part II: Influence of Process Parameters. AAPS PharmSciTech 2009;10:1040-7.

52. Jin X, Zhang Z, Sun E, Tan X, Li S, Cheng X, et al. Enhanced oral absorption of 20(S)-protopanaxadiol by self-assembled liquid crystalline nanoparticles containing piperine: in vitro and in vivo studies. Int J Nanomedicine 2013;8:641-52.

53. Zhiwen Y, Yinhe T, Meiwan C, Linghui D, Ziyun S, Xinsheng $\mathrm{P}$, et al. Development of Amphotericin B-Loaded Cubosomes Through the SolEmuls Technology for Enhancing the Oral Bioavailability. AAPS Pharm SciTech 2012;13(4):1483-91.

54. Jain S, Rathi VV, Jain AK, Das M, Godugu C. Folatedecorated PLGA nanoparticles as a rationally designed vehicle for the oral delivery of insulin. Nanomedicine (Lond) 2012;7(9):1311-37.

55. Nguyen TH, Hanley T, Porter CJ, Boyd BJ. Nanostructured liquid crystalline particles provide long duration sustainedrelease effect for a poorly water soluble drug after oral administration. J Control Release 2011;153(2):180-6.

56. Thapa RK, Baskaran R, Madheswaran T, Rhyu JY, Kim JO, Yong CS, et al. Effect of saturated fatty acids on tacrolimusloaded liquid crystalline nanoparticles. J Drug Deliv Sci Technol 2013;23(2):137-41.

57. Lee KWY, Nguyen TH, Hanley T, Boyd BJ. Nanostructure of liquid crystalline matrix determines in vitro sustained release and in vivo oral absorption kinetics for hydrophilic model drugs. Int J Pharm 2009;365(1-2):190-9.

58. Esposito E, Cortesi R, Drechsler M, Paccamiccio L, Mariani $\mathrm{P}$, Contado $\mathrm{C}$, et al. Cubosome dispersions as delivery systems for percutaneous administration of indomethacin. Pharm Res 2005;22(12):2163-73.

59. Chen Y, Lu Y, Zhong Y, Wang Q, Wu W, Gao S. Ocular delivery of cyclosporine A based on glyceryl monooleate/ poloxamer 407 liquid crystalline nanoparticles: preparation, characterization, in vitro corneal penetration and ocular irritation. J Drug Target 2012;20(10):856-63.

60. Sadhale Y, Shah JC. Stabilization of insulin against agitationinduced aggregation by the GMO cubic phase gel. Int J Pharm 1999;1919(1):51-64.

61. Sadhale Y, Shah JC. Biological activity of insulin in GMO gels and the effect of agitation. Int J Pharm 1999;191(1):65-74.
62. Sadhale Y, Shah JC. Glyceryl monooleate cubic phase gel as chemical stability enhancer of cefazolin and cefuroxime. Pharm Dev Technol 1998;3(4):549-56.

63. Spillmann CM, Naciri J, Algar WR, Medintz IL, Delehanty JB. Multifunctional liquid crystal nanoparticles for intracellular fluorescent imaging and drug delivery. ACS Nano 2014;8(7):6986-97.

64. Nilsson C, Barrios-Lopez B, Kallinen A, Laurinmäki P, Butcher SJ, Raki M, et al. SPECT/CT imaging of radiolabeled cubosomes and hexosomes for potential the ranostic applications. Biomaterials 2013;34(33):8491-503.

65. Boyd BJ, Khoo SM, Whittake DV, Davey G, Porter CJH. A lipid-based liquid crystalline matrix that provides sustained release and enhanced oral bioavailability for a model poorly water soluble drug in rats. Int J Pharm 2007;340(1-2):52-60.

66. Boyd BJ, Whittaker DV, Khoo SM, Davey G. Hexosomes formed from glycerate surfactants-formulation as a colloidal carrier for irinotecan. Int J Pharm 2006;318(1-2):154-62.

67. Lopes LB, Speretta FFF, Bentley MV. Enhancement of skin penetration of vitamin $\mathrm{K}$ using monoolein-based liquid crystalline systems. Eur J Pharm Sci 2007;32(3):209-15.

68. Swarnakar NK, Jain V, Dubey V, Mishra D, Jain NK. Enhanced oromucosal delivery of progesterone via hexosomes. Pharm Res 2007;24(12):2223-30.

69. Fong WK, Hanley T, Boyd BJ. Stimuli responsive liquid crystals provide 'on-demand' drug delivery in vitro and in vivo. J Control Release 2009;135(3):218-26.

70. Lee DR, Park JS, Bae IH, Lee Y, Kim BM. Liquid crystal nanoparticle formulation as an oral drug delivery system for liver-specific distribution. Int $J$ Nanomedicine 2016;11:853-71.

71. Swarnakar NK, Thanki K, Jain S. Lyotropic liquid crystalline nanoparticles of coq10: implication of lipase digestibility on oral bioavailability, in vivo antioxidant activity, and in vitro in vivo relationships. Mol Pharm 2014;11(5):1435-49.

72. Okawara M, Hashimoto F, Todo H, Sugibayashi K, Tokudome Y. Effect of liquid crystals with cyclodextrin on the bioavailability of a poorly water-soluble compound, diosgenin, after its oral administration to rats. Int J Pharm 2014;472(1):257-61.

73. Yaghmur A, Kriechbaum M, Amenitsch H, Steinhart M, Laggner P, Rappolt M. Effects of pressure and temperature on the self-assembled fully hydrated nanostructures of monoolein-oil systems. Langmuir 2010;26(2):1177-85.

74. Sallam AS, Khalil E, Ibrahim H, Freij I. Formulation of an oral dosage form utilizing the properties of cubic liquid crystalline phases of glyceryl monooleate. Eur J Pharm Biopharm 2002;53(3):343-52.

75. Negrini R. Mezzenga R.pH-responsive lyotropic liquid crystals for controlled drug delivery. Langmuir 2011;27(9):5296-303.

76. Negrini R, Fong WK, Boyd BJ, Mezzenga R. pH-Responsive lyotropic liquid crystals and their potential therapeutic role in cancer treatment. Chem Commun 2015;51(30):6671-74.

77. Yaghmur A, Larsen SW, Schmitt M, Østergaard J, Larsen C, Jensen $\mathrm{H}$, et al. In situ characterization of lipidic bupivacaineloaded formulations. Soft Matter 2011;7(18):8291-95.

78. Yaghmur A, Rappolt M, Oostergaard J, Larsen C, Larsen SW. Characterization of bupivacaine-loaded formulations based on liquid crystalline phases and micro emulsions: the effect of lipid composition. Langmuir 2012;28(5):2881-9.

79. Yuli A, Garti N. Transitions induced by solubilized fat into reverse hexagonal mesophases. Colloids Surf B Biointerfaces 2005;43(2):72-82. 